\title{
Preputial Calculus in a Neurologically-impaired Child
}

\author{
RI SPATARU, DA IozSA AND M IVANOV \\ From Department of Pediatric Surgery, Emergency Clinical Hospital for Children “Maria Sklodowska Curie”, University of \\ Medicine and Pharmacy “Carol Davila” Bucharest, Romania.
}

Correspondence to:

Dr Spataru Radu-Iulian: 20, Bld.

Brancoveanu C., PC 041451,

Emergency Clinical Hospital for Children

“Maria Sklodowska Curie”, Bucharest,

Romania,radu_spataru@yahoo.com

Received: July 28, 2014;

Initial review: September 01, 2014;

Accepted: October 09, 2014.

\begin{abstract}
Background: Preputial calculi are rarely encountered in childhood. Case characteristics: A 5-year-old boy with symptoms of chronic balanoposthitis. Observation: A preputial stone was documented and removed at circumcision. Outcome: Uneventful postoperative recovery. Message: In children, association between phimosis and neurologic impairment represent predisposing condition for preputial stone formation.
\end{abstract}

Keywords: Children, Phimosis, Prepuce.
$\mathrm{P}$ reputial calculi are an uncommon entity. It has been described in isolated case reports, mostly in elderly men, with tight phimosis and poor hygiene [1-4]. It is postulated that these calculi originate from inspissated smegma with lime salts trapped into the phimotic prepuce, infected stagnant urine or migrated calculi from the upper urinary tract into the preputial sac [1]. Preputial calculi in children are very rare [5-7] , usually associated with phimosis and other urologic and/or neurologic anomalies.

\section{CASE RePORT}

A 5-year-old-male was referred to our hospital with the presumed diagnosis of balanoposthitis. The child had paraparesis and incontinence for urine and feces, following surgery in infancy for myelomeningocele. The penis looked severely swollen, from the tip almost to the penoscrotal junction. The foreskin was significantly thickened, with a tight phimotic ring showing ulceration on its circumference, making impossible examination of the meatus opening (Fig. 1a). No stricture band or hair tourniquet could be found along the penile shaft or at the base of the penis. Testes and scrotum looked normal. Significant diaper rash and pressure sores were evident in the inguinal, perineal and sacral area. No signs of acute urine retention were noted. The patient presented intermittent leaking urine without an obvious stream. The patient was diagnosed with chronic balanoposthitis probably occurring in the context of neurological impairment and tight phimosis. Bladder catheterization for urine analysis was unsuccessful. A metal probe was attempted to pass through the foreskin opening when a presumable foreign body was identified with a hard, stone-like consistency.
The radiologic examination of the abdomen and pelvis showed a well-defined, round, radiopaque shadow in the distal penile region. Ultrasound revealed a bright hyperechoic structure located in the preputial sac; there were no signs of urolithiasis.

Circumcision was performed (Fig. 1 b-d) with the removal of a thick preputial sac molded on a $3 / 2 \mathrm{~cm}$ ovalar calculus with the glans imprint on it, light colored with a rough surface. Penis had a normal size with an adequate meatus opening. Postop urethral catheterization showed presence of leukocyte, but negative urine culture. Postoperative recovery was uneventful. Chemical analysis of the stone showed calcium oxalate with no foreign bodies found inside. Serum and urinary levels of electrolytes and uric acid were in normal ranges; parathyroid hormone level was also normal.

Pathological exam of the prepuce confirmed balanoposthitis.

\section{Discussion}

In the first years of life the prepuce is attached to the glans by physiological congenital adhesions, so there is a little room for urine to collect in the preputial sac. There have been described, in extremely rare cases, congenital giant deep preputial sac with very large capacity for urine collection [8], which could favor stone formation. In our case, no such anomaly could be identified.

Scarring of the prepuce with progressive fibrous phimosis due to chronic pathological conditions such as Balanitis xerotica obliterans (BXO) usually occurs in older children and adults. Newly published data has shown a higher incidence than was previously reported in 


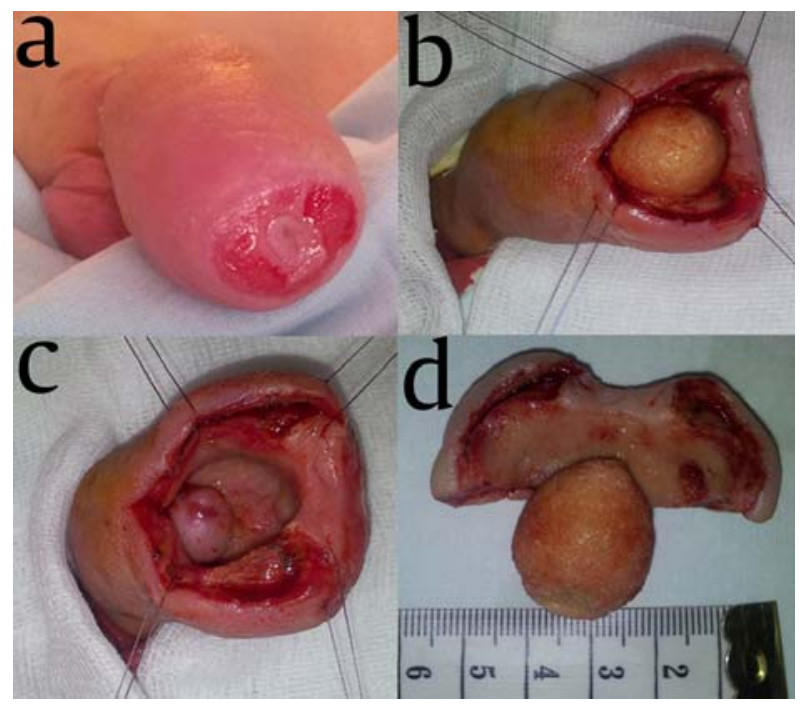

FIG. 1 (a) Penis severely swollen, from the tip almost to the penoscrotal junction; tight phimotic ring showing ulceration on its circumference; $(b, c, d)$ removal of a thick preputial sac molded on a $3 / 2 \mathrm{~cm}$ ovalar calculus with the glans imprint on it, light colored with a rough surface. (See color image at website)

children under the age of 5 , if the diagnosis is based on histopathological examination at the time of circumcision [9]. Our histopathological examination was able to exclude $\mathrm{BXO}$ as a possible cause for phimosis.

In adults with neurogenic bladder, $62.5 \%$ of nephroliths were metabolic disease-related [10]. This research highlights the importance of documenting metabolic disorders in neurologically-impaired patients with urolithiasis. In children, preputial stones occur in the presence of phimosis associated with other urological and/or neurological malformation (e.g. epispadias and low imperforated anus and myelomeningocele). Neglected preputial stones may generate serious complications such as bilateral hydronephrosis and acute renal failure [1], or even penile carcinoma [3]. One case described in a child showed preputial skin fistula as a consequence of multiple preputial calculi [7].

In our case, evaluation excluded presence of calculi anywhere else along the urinary tract, or foreign body as a nucleus, or signs of present urinary infection. We can assume that the possible etiology was either migration of a proximal situated micro-calculus, which developed further in the tight preputial sac or de novo formation of the calculus in the stagnant urine in the preputial cavity.

In conclusion, preputial calculi can occur in childhood, association between tight phimosis and urinary incontinence representing a predisposing circumstance.

Contributors: SR: Conception of the work; revising paper for important intellectual content; IDA: Analysis and interpretation of the data for the work; drafting the work; IM: Acquisition, analysis and interpretation of the data for the work; drafting the work and revising it critically for important intellectual content. All authors approved the final manuscript and take responsibility for its conent.

Funding: None; Competing interests: None stated.

\section{REFERENCES}

1. Yuasa T, Kageyama S, Yoshiki T, Okada Y. Preputial calculi: a case report. Acta Urol Jpn. 2001;47:513-5.

2. Hori N, Hoshina A, Kinoshita N, Kato M, Arima K, Tajima K, et al. A case of preputial calculi. Acta Urol Jpn. 1985;31:327-9.

3. Mohapatra TP, Kumar S. Concurrent preputial calculi and penile carcinoma - a rare association. Postgrad Med J. 1989;65:256-7.

4. Nagata D, Sasaki S, Umemoto Y, Kohri K. Preputial calculi. BJU Int. 1999;83:1076-7.

5. Sharma SK. Phimosis and the preputial calculus. Indian Pediatr. 1983;20:386.

6. Ellis DJ, Siegel AL, Elder JS, Duckett JW. Preputial calculus: a case report. J Urol. 1986; 136:464-5.

7. Tuðlu D, Yuvanç E, Yilmaz E, Batislam E, Gürer YKY. Unknown complication of preputial calculi: preputial skin fistula. Int Urol Nephrol, 2013, July 25, online, DOI 10.1007/s11255-013-0496-x.

8. Philip I, Hull NJL. Congenital giant prepucial sac: Case reports. J Pediatr Surg. 1999;3: 507-8.

9. Jayakumar S, Antao B, Bevington O, Furness P, Ninan GK. Balanitis xerotica obliterans in children and its incidence under the age of 5 years. J Pediatr Urol. 2012;8:272-5.

10. Matlaga BR, Kim SC, Watkins SL, Kuo RL, Munch LC, Lingeman JE. Changing composition of renal calculi in patients with neurogenic bladder. J Urol. 2006;175: 1716-9. 